\title{
Del plan a la política de creación de nuevas universidades nacionales en Argentina: la expansión institucional de los años 70 revisitada Laura Rovelli
}

Laura Rovelli es Licenciada en Ciencia Política (UBA) y docente de la UNLP. Magíster en Ciencias Sociales (FLACSO- Argentina) y doctoranda por la Facultad de Ciencias Sociales (UBA-CONICET).

Mail: laurarovelli@gmail.com

\section{resumen}

El presente artículo analiza los circuitos de producción de la "idea" de creación de nuevas universidades nacionales entre 1971 y 1973 en Argentina. Para ello, indaga el recorrido de las ideas por el espacio burocrático-estatal y académico-universitario y sus entrecruzamientos, los que dieron origen a un conjunto de reglas, normas y saberes especializados cuyos contenidos impusieron y permitieron prácticas específicas para su implementación. Siguiendo esa línea, examina los modelos universitarios que predominaron en los circuitos de expertos e identifica cuáles se incorporaron finalmente a las estrategias gubernamentales.

El estudio se vale de la idea de que en paralelo a las estrategias represivas del gobierno autoritario, algunos expertos idearon otros modelos universitarios que pudieran contener el avance de la contestación social y la deslegitimación estatal. Los intentos se concretaron en distintos planes técnicos, dieron lugar a numerosas estructuras burocráticas y moldearon las reglas y las dinámicas institucionales de las noveles universidades. Por otra parte, el proceso de creación de nuevas universidades nacionales de los años 70 inauguró una racionalidad de expansión institucional mediante la cual el poder político buscó imponer a las casas de estudio un formato diferente, en oposición al modelo político y académico de las universidades tradicionales.

\section{palabras claves}

universidad / autoritarismo / modernización / expertos / Estado

\section{summary}

This research analyses the circuits of production of the "idea" of new national universities between 1971 and 1973 in Argentina. It probes into the itineraries of those ideas into the state-bureaucratic and academic space and their criss-crosses, which produced a set of rules, norms and specialized knowledges along with the imposition and allowance of specific practices for their enforcement. In addition, it examines which higher education models predominated in expertise circuits and it identifies the ones that were finally incorporated in the frame of governmental strategies.

The study introduces the argument that together with repressive governmental strategies, some experts devised other models of higher education that could content the advance of social protest and state illegitimacy. Those attempts moulded different technical plans, promoted numerous bureaucratic structures and shaped the rules and institutional dynamics of the novel universities. In addition, by means of the creation of new universities in the 70, the government introduced a rationality of institutional expansion which sought to impose a different higher educational format, in opposition to the one of traditional universities.

\section{keywords}

university / authoritarism / modernization / experts / State 


\section{Presentación}

Entre 1971 y 1973 surgieron trece nuevas universidades mediante la fundación de nuevas casas de estudio, la subdivisión de instituciones preexistentes o la nacionalización de anteriores establecimientos privados o provinciales. ${ }^{1}$ En los siguientes siete años, y como resultado de los proyectos inconclusos del período anterior, se nacionalizaron otras tres, con lo que quedó clausurada la etapa de mayor expansión universitaria del país. ${ }^{2}$

Los avatares de esas nuevas instituciones no se apartaron de la dinámica histórica propia de las universidades nacionales, la que se desarrolló en estrecha vinculación con los ciclos políticos locales. En efecto, las universidades argentinas fueron durante décadas objeto y arena de múltiples luchas políticas. No resulta extraño entonces que, de manera general, las distintas épocas institucionales se desarrollaran en consonancia con los vaivenes políticos. ${ }^{3}$ Pero también, por su carácter público se vieron afectadas por los cambios de gobierno y las transformaciones en el Estado. De tal forma, la fragilidad de esas instituciones puede ser analizada como una manifestación más de la debilidad estatal y, concretamente, de los conflictos y particularidades de sus estructuras burocráticas (Sigal, 2002: 50).

El análisis aquí desarrollado, antes de explicar la política universitaria desde la revisión crítica de la relación Estado-sociedad (Pérez Lindo, 1985), los procesos de modernización educativa (Bertoni y Cano, 1990; Chiroleu, 2004 y Krotsch, 2001) o la historia de las universidades argentinas (Buchdinder, 2005), pretende destacar la dinámica institucional de las ideas y los saberes especializados que dieron forma a la toma de decisiones políticas en favor de la creación de nuevas casas de estudio. Siguiendo esa línea, el marco analítico de la investigación se beneficia de algunos trabajos de la corriente teórica "neoinstitucionalista" (Skocpol y Veir, 1993; Sikkink, 1991), la que, en líneas generales, propone indagar "las ideas en las políticas"; vale decir, las nociones que orientan las acciones concretas de los gobiernos. Este enfoque, también plantea el "regreso del Estado" a un primer plano (Evans, Rueschemeyer y Skocpol, 1985), cuestión que analiza a través de las políticas públicas, centrándose en la estructura, naturaleza y dinámica estatal.

Por otra parte, al considerar la perspectiva histórica en las ciencias sociales (Skocpol, 1984 y Wagner et al., 1999; entre otros), el escrito propone rastrear algunos de los itinerarios que, en el horizonte de los interrogantes y los conflictos de la época, transformaron esas ideas en decisiones políticas. De tal forma, se procura analizar en un tiempo histórico acelerado por la coyuntura sociopolítica, los modelos universitarios que predominaron en los circuitos de expertos e identificar cuáles se incorporaron finalmente a las estrategias gubernamentales para hacer frente a la crisis social y estatal. ${ }^{4}$ Tomamos en cuenta la idea de "revolución" -tan expandida en el campo social, entre los cuales el universitario fue uno de los más destacados ${ }^{5}$ - que también formó parte de las previsiones de expertos y políticos conservadores.

Para abordar el problema de investigación se adoptó una estrategia metodológica cualitativa, la que se valió de distintas técnicas de pesquisa: relevamiento de fuentes bibliográficas y documentales, junto con la realización de entrevistas 
en profundidad a personalidades que intervinieron en el proceso de toma de decisiones en el espacio estatal de esos años. Se entrevistó a todos los ministros de Educación y Cultura de la autodenominada Revolución Argentina ${ }^{6}$ : Dardo Pérez Guilhou, José Luis Cantini y Gustavo Malek. También se efectuaron entrevistas en profundidad a dos figuras relevantes: Alberto C. Taquini (h), el principal ideólogo del Plan de Nuevas Universidades, y a Antonio Salonia, quien participó en un breve período (entre 1969 y 1970) de los equipos técnicos del Ministerio de Cultura y Educación. No obstante, con el propósito de reducir y enmendar posibles olvidos, malentendidos, recuerdos parciales o distorsionados de los propios relatos, consideramos oportuno contextualizar esos testimonios con documentos y material de archivo (en especial, diarios y revistas de la época).

El presente estudio se vale de la idea de que en paralelo a las estrategias represivas del gobierno autoritario, algunos sectores idearon otros modelos universitarios que pudieran contener el avance de la contestación social y la deslegitimación estatal. En ese marco, se retomaron algunas ideas y proyectos universitarios inconclusos del período 1955-1966, en especial aquellos ligados a la idea de "universidad para el desarrollo", mientras que las universidades tradicionales comenzaron a ser tematizadas en términos de "sobredimensionamiento" e incluso "monstruosidad". Esos intentos se concretaron en distintos planes técnicos, dieron lugar a numerosas estructuras burocráticas y moldearon en parte las reglas y las dinámicas institucionales de las noveles universidades. Entre ellos, la investigación plantea que el Plan de Nuevas Universidades, más conocido como "El Plan Taquini", buscó "descongestionar" la matrícula de las universidades metropolitanas hacia instituciones que funcionaran como satélite de aquellas, subsumiendo la idea de desarrollo a la de seguridad. Por su parte, la política de expansión universitaria del gobierno de Lanusse, aunque fuertemente influida por esa propuesta técnicointelectual, amplió los alcances de la expansión institucional en el contexto de una coyuntura política crítica y de la necesidad del régimen de contar con apoyos político-institucionales.

En el mediano plazo, el proceso de creación de nuevas universidades nacionales de los años 70 inauguró una dinámica de expansión institucional mediante la cual el poder político buscó imponer a las casas de estudio un formato diferente, en oposición al modelo político y académico-institucional de las universidades tradicionales. Por tanto, no resulta extraño que en los momentos de mayor tensión y/o enfrentamiento entre éstas y el Estado nacional, se levantaran los puentes para habilitar el surgimiento de nuevas instituciones. Finalmente, la circulación de expertos por las fronteras estatales, en particular en el segmento de políticas universitarias, resultó favorable a la innovación intelectual, la cual daría origen a una nueva idea de universidad y favorecería la creación de nuevas instituciones. En cierta medida, el hecho de que la política de expansión universitaria de los años 70 sea también conocida como el "Plan Taquini" revela la importancia que los expertos alcanzaron en ese proceso. 


\section{Gobernar a la universidad: intervención, reglamentación y modernización al servicio de la seguridad}

En 1966, un nuevo golpe de Estado proclamó la transformación nacional y la modernización de Argentina a través del disciplinamiento de la sociedad. Los distintos sectores que convergieron en el apoyo a la Junta Militar Revolucionaria -en particular liberales y nacionalistas- pretendieron reconfigurar la relación entre la sociedad y el Estado (Cavarozzi, 1983: 11; Quiroga, 2004: 42). Para ello, acordaron impugnar el sistema político semidemocrático vigente junto con cualquier otra expresión política, por considerarlos factores de inestabilidad y desorden que podrían atentar contra la reestructuración económica a implantar. A partir de marzo de 1967, el imperativo de la política económica implementada por el nuevo ministro de Economía, Adalbert Krieger Vasena, consistió en disminuir drásticamente la inflación por medio del ajuste global de la economía, el cual congeló la participación de los sectores populares en la distribución de los ingresos.

Las expectativas por lograr el "despegue" de un país que, como Argentina, se caracterizaba por el estancamiento económico, mudaron desde las formas tradicionales de gestión de la democracia a la adopción de las vías revolucionarias. En efecto, la revolución resultó ser ampliamente aceptada en el clima de ideas de la época, tanto por sectores de izquierda como de derecha, considerándola la única salida para alcanzar esos objetivos. Así, estos últimos eliminaron el Congreso Nacional, apartaron a los jueces de la Corte Suprema de sus cargos, suprimieron las elecciones e inhabilitaron a los partidos políticos para ejercer sus actividades. Asimismo, desmantelaron numerosos circuitos culturales, profesionales, técnicos y académicos por un período indefinido.

Por otra parte, la recuperación de cierto ideario desarrollista, junto con la difusión de la hipótesis de la Doctrina de la Seguridad Nacional -según la cual los focos de agresión interna o de subversión constituían una nueva forma de conspiración marxista internacional- sentaron las bases de la experiencia autoritaria en torno de la cuestión del "desarrollo" y la "seguridad". Si bien esa doctrina constituyó el sustento ideológico de las dictaduras institucionales de las Fuerzas Armadas latinoamericanas, conviene destacar que en el caso de Brasil el foco fue puesto en la relación entre desarrollo económico y seguridad interna y externa, a tal punto que fue conocida como la Doutrina de Segurança e Desenvolvimento (Seguridad y Desarrollo) (Ansaldi, 2004: 45). En contraste, la dictadura argentina de 1966-1973 basó su legitimidad en la promesa de reestablecer un orden político estable y cumplir con el proceso de modernización iniciado por el desarrollismo, pero subsumiendo este último objetivo a la cuestión de la seguridad.

En ese marco, las universidades nacionales se convirtieron en el centro de la oposición pública al nuevo régimen. El proceso de modernización que venía desarrollándose desde finales de la década de 1950 en el campo intelectual y cultural, favoreció ese posicionamiento. En efecto, en esos años las universidades nacionales se configuraron como lugares significativos para debatir e impulsar proyectos modernizadores, pero también para redefinir su función política y social. Con todo, muchas de esas iniciativas se desarrollaron por la agregación o por superposición 
de sedimentos diferentes (Naishtat, et al., 2005), lo que generó un abigarramiento y una amplia heterogeneidad institucional en la universidad primero, y en el sistema nacional universitario después. Otras, quedaron inconclusas aunque algunas ideas-fuerza operaron como telón de fondo de los intentos modernizadores de la autodenominada Revolución Argentina. De todas formas, si durante el período desarrollista las universidades habían sido concebidas como "motores del desarrollo", hacia finales del período y en particular con el nuevo régimen autoritario, se las identificó con los "focos de perturbación pública"?

En julio de 1966, a través de su intervención, se dispuso la primera acción para regular a las universidades. En la Universidad de Buenos Aires (Decreto-Ley 16.912), la medida provocó los hechos de violencia conocidos como "la noche de los bastones largos" y renuncia de muchos profesores, principalmente de las Facultades de Ciencias Exactas y de Filosofía y Letras. No obstante, el impacto de la intervención en otras instituciones ha sido frecuentemente opacado en la literatura especializada por la experiencia de Buenos Aires. ${ }^{8}$ En ese sentido, una investigación reciente reveló que en el caso de la Universidad Nacional de La Plata, la intervención no impidió que en algunas carreras, como Ciencias de la Educación, el proyecto reformista del período 1955-1966 pudiera continuarse y hasta incluso profundizarse (Suasnábar, 2004).

De todas formas, la expulsión de académicos por motivos ideológicos -aunque todavía no se conoce en profundidad la magnitud institucional y disciplinar del mismo- originó un proceso de migración forzada hacia los nuevos institutos universitarios, las universidades privadas y provinciales e incluso a distintos circuitos académicos del exterior. ${ }^{9}$ La "fuga de cerebros" (brain drain), tal como se tematizó en la prensa y en la literatura científica de la época, fue considerada uno de los principales obstáculos para las estrategias de desarrollo.

La Ley de Ministerios № 16.953 sancionada por el gobierno militar de 1966 otorgó competencias sobre la cultura y la educación a la Secretaría de Estado con ese mismo nombre pero bajo la jurisdicción del Ministerio del Interior. ${ }^{10}$ La subordinación de la esfera educativa a la racionalidad de otras agencias estatales, como la del Ministerio de Economía y del Interior, expresó las líneas de fuerza más importantes en el proceso de gestación y construcción de las políticas públicas durante ese primer período del gobierno militar.

Un año más tarde, se intentaría alcanzar la normalización de las casas de estudio a través de una nueva Ley Orgánica de Universidades (17.245/67), firmada por el presidente de facto Onganía y los ministros del Interior y Economía, Guillermo Borda y Adalberto Krieger Vasena, respectivamente. La misma eliminó la posibilidad de cualquier actividad política y reformuló el gobierno universitario a través de la supresión del modelo tripartito y la imposición del principio de la jerarquía académica de los profesores.

Además, la normativa estableció que el Consejo de Rectores debería coordinar en todo el país las condiciones de admisión a las diversas carreras (art. 81). Asociado a la reforma administrativa recomendada para las universidades latinoamericanas de la época, se esperaba que la conformación de esa institución permitiera 
[...] una organización seria y científica del planeamiento universitario y de la promoción de todos aquellos proyectos que posibilitaran la reformulación estructural de la educación superior [...] (Atcon, 1966: 118).

A la vez, se introdujo la aprobación de pruebas de ingreso reglamentadas por cada Facultad como requisito de admisión a las universidades (art. 82). En este sentido, corresponde señalar que a partir de 1957 comenzó a desplegarse una política restrictiva de acceso a la universidad a través de cursos de ingreso con diversas modalidades de evaluación o, directamente, mediante exámenes eliminatorios. Sin embargo, recién en 1970 las autoridades de la dictadura institucional llevaron a la práctica los principios de la ley $17.245 \mathrm{al}$ implementar un sistema de ingreso en distintas Facultades de las universidades públicas, incluso en aquellas que no contaban anteriormente con ese régimen. En esos años, la "lucha contra el limitacionismo" se transformó en una de las consignas más importantes del movimiento estudiantil.

Sumado a lo anterior, la reglamentación mantuvo la gratuidad de la enseñanza pero sólo en el grado y para aquellos estudiantes que alcanzaran un mínimo de materias aprobadas (art. 92). Paralelamente, recogió algunos principios de los sectores modernizadores del período previo, referidos a la organización de las universidades. En esa línea, contempló la estructura departamental (art. 12) y la posibilidad de organizar las carreras en ciclos, al final de los cuales se otorgarían los certificados correspondientes (art. 85).

Por su parte, las nuevas universidades privadas y provinciales tampoco quedaron exentas de los intentos del régimen autoritario por direccionar la modernización de la educación superior y regular su proceso de expansión, como resultado de la reglamentación del célebre artículo 28 del decreto 6.403/55 (ratificado por la ley 14.557 durante el gobierno de Frondizi). Frente a la amplia variedad de instituciones y a la alta disparidad de ofertas educativas, a partir de las leyes 17.604/67 y 17.778/68 respectivamente, el Estado nacional se reservó la capacidad de habilitación de los títulos de las nuevas instituciones de enseñanza universitaria provincial y privada. ${ }^{11}$ No obstante, en el caso de estas últimas, la normativa disminuyó de hecho la regulación estatal, al extender a las universidades privadas con más de quince años de funcionamiento la habilitación para el otorgamiento de títulos profesionales sin necesidad de contralor por parte del Estado.

La autodenominada Revolución Argentina también procuró recrear ciertas estructuras estatales establecidas durante el período desarrollista. En ese sentido, el Consejo Nacional de Planeamiento (CONADE), agencia estatal creada durante el gobierno de Frondizi, se mantuvo en el mapa institucional del Estado, aunque pasó a formar parte -junto con el Consejo Nacional de Seguridad (CONASE) y el Consejo Nacional de Ciencia y Técnica (CONACYT) - del "Sistema Nacional de Planeamiento y Acción para la Seguridad Nacional".

En el plano estrictamente universitario, la creación de la Dirección Nacional de Altos Estudios en 1968 reformuló y expandió la estructura de la anterior Inspección General de Enseñanza Universitaria Privada, surgida en 1959. La nueva dirección tuvo a su cargo la supervisión de las actividades de las nuevas casas de 
estudio (aquellas con menos de quince años de funcionamiento), a través de la autorización de los títulos habilitantes, la evaluación de la calidad de los egresados y de diversos estudios de prefactibilidad para lograr la aprobación de las nuevas instituciones privadas y provinciales.

Del análisis de las regulaciones, acciones y estructuras estatales detalladas anteriormente resulta posible distinguir en el período dos estrategias de gobierno de la universidad: una, intensamente represiva; otra, de corte modernizador y tecnocrático. ${ }^{12}$ La primera eliminó la autonomía universitaria, desmanteló numerosos grupos de investigación en el interior de varias Facultades y procuró obturar el acceso a los estudiantes. La segunda intentó promover, a través de la nueva normativa universitaria, la adopción de cambios en las estructuras y en la organización de las instituciones más acordes con los modelos de la época. También pretendió fijar mecanismos de regulación y coordinación de las nuevas casas de estudio, los cuales se diluyeron frente al ejercicio centralizado y autoritario del poder. Finalmente y como parte de esta segunda tendencia, se incorporaron a la esfera educativa del Estado diversos especialistas en carácter de técnicos.

\section{El ascenso de la problemática universitaria de la mano de los especialistas}

El llamado Cordobazo, de mayo de 1969, constituyó un punto de quiebre en la política del régimen autoritario. Esos hechos señalaron el inicio del fin del gobierno de Onganía, al mostrar la magnitud del descontento social, el deterioro de la política económica de Krieger Vasena y el fracaso de la dictadura institucional para superar la crisis social y estatal que había comenzado con el derrocamiento y posterior proscripción del peronismo. Por otra parte, adquirieron un amplio efecto de demostración para otros sectores sociales, dado que en adelante proliferaron los alzamientos populares; las huelgas y la protesta estudiantil crecieron enérgicamente en las universidades (De Riz, 2000: 75), mientras se radicalizaron los reclamos frente a la actitud defensiva del gobierno.

Algunos meses después de la revuelta obrero-estudiantil, y como resultado del ascenso de lo educativo dentro de las cuestiones apremiantes por su problematización social, una nueva Ley de Ministerios ( $N^{\circ}$ 183.416, sancionada el 20/10/69) devolvió al área su jerarquía ministerial. El Ministerio de Cultura y Educación contó con dos subsecretarías en esas áreas, aunque la segunda absorbió la mayor parte del presupuesto y de los especialistas del sector. Esta transformación del aparato del Estado puede entenderse simultáneamente como un reflejo de las relaciones que se establecieron en la sociedad, pero también como un nuevo espacio desde donde se intentarían ejecutar las medidas necesarias para recomponer el orden social.

Una nueva gestión educativa, encabezada por el rector de la Universidad de Cuyo, Dardo Pérez Guilhou, combinó la continuidad y el cambio de los funcionarios del área: a Alfredo Van Gelderen y Luis Jorge Zanotti se agregaron entre otros, Antonio Salonia y Gustavo Cirigliano. Así, una nueva generación de intelectuales católicos destacados en la especialidad educativa, como fue el caso de Emilio F. 
Mignone, cobraron protagonismo en el proceso de toma de decisiones. Muchos de ellos se formaron en distintos campos de las ciencias sociales, obtuvieron becas y circularon por organismos nacionales y/o internacionales de planificación. ${ }^{13}$

Por fuera del Estado, aunque con amplias redes de relaciones con miembros del gobierno, un grupo de personalidades -nucleadas en torno a la figura de Alberto C. Taquini- plantearon hacia 1967 el crecimiento de la población estudiantil universitaria como uno de los problemas socialmente más relevantes. ${ }^{14} \mathrm{~A}$ partir del diagnóstico de la existencia de una "superpoblación estudiantil" (Taquini, et al., 1972: 132) y de "universidades multitudinarias" (pp.138), postulaban [...] la urgente necesidad de planificar la política a seguir durante los próximos años, a fin de asegurar la mayor eficiencia del sistema operativo de las universidades, actualmente distorsionado por la inadecuada relación del número de universidades con el de estudiantes (Ibídem).

En noviembre de 1968 se presentó públicamente un plan-concebido como una "idea de política educacional" (pp.135)- de fundación de universidades nacionales en distintas regiones del país. En efecto, el "Programa de Adecuamiento de la enseñanza Universitaria Argentina a las Necesidades del Desarrollo" fue presentado en el Coloquio sobre Modernización de las Instituciones Políticas en la Argentina, organizado por la Academia del Plata en la localidad de Chilecito, provincia de La Rioja. El mismo se expuso en un lugar con una fuerza simbólica muy fuerte: la finca de Joaquín V. González en Samay-Huasi.

No obstante, conviene señalar que hasta ese momento en anteriores planes e informes de gobierno el aumento de la población estudiantil no había constituido un tema de política; por el contrario, su incremento había sido evaluado positivamente por parte de diversos expertos. Es más, las preocupaciones centrales referidas a la educación universitaria giraban en torno a la distribución de la matrícula (excesivamente direccionada hacia carreras tradicionales como Abogacía, Medicina e Ingeniería) y a los bajos porcentajes de graduación, por lo que la creación de nuevas universidades no formaba parte de la "agenda de controversia pública". ${ }^{15}$ Así lo demuestran los registros del Plan Nacional de Desarrollo (1965-1969), iniciado durante la presidencia de Arturo Illia y elaborado por el Consejo Nacional de Desarrollo (CONADE), como también el célebre diagnóstico-informe sobre el sector educativo, iniciado en esos años por el CONADE con el asesoramiento de una misión de la Organización de Cooperación y Desarrollo Económico (OCDE) encabezada por el especialista Louis Emmerij.

Hacia 1969, la población universitaria llegaba a 238.000 alumnos, cifra que había sido calculada por la OCDE para 1974 (Taquini, 1970: 435-436). Las estimaciones realizadas por el grupo de Taquini sostenían que la población universitaria superaría para 1980 la cifra difundida por la OCDE alcanzando los 350.000 estudiantes, es decir habría 60.000 inscriptos más de lo que señalaba el primer informe (Ibídem: 438). Finalmente, y a pesar de los vaivenes político-militares y de las distintas limitaciones al ingreso, la matrícula universitaria aumentó, en efecto, más de lo proyectado por el informe de la OCDE, sin llegar a la cifra pronosticada por el grupo Taquini para 1980 (al respecto, ver cuadro I). 
Cuadro I. Evolución de la matrícula universitaria 1955-1980

\begin{tabular}{|r|r|r|r|}
\hline Años & Nacional & Privado & $\begin{array}{c}\text { Total } \\
\text { Universitario }\end{array}$ \\
\hline 1955 & 136.362 & ---- & $\mathbf{1 3 6 . 3 6 2}$ \\
\hline 1956 & 132.557 & ---- & $\mathbf{1 3 2 . 5 5 7}$ \\
\hline 1957 & 142.959 & ---- & $\mathbf{1 4 2 . 9 5 9}$ \\
\hline 1958 & 137.673 & 1.536 & $\mathbf{1 3 9 . 2 0 9}$ \\
\hline 1959 & 146.937 & 2.120 & $\mathbf{1 4 9 . 0 5 7}$ \\
\hline 1960 & 157.163 & 3.884 & $\mathbf{1 6 1 . 0 4 7}$ \\
\hline 1961 & 155.568 & 5.290 & $\mathbf{1 6 0 . 8 5 8}$ \\
\hline 1962 & 161.263 & 6.898 & $\mathbf{1 6 8 . 1 6 1}$ \\
\hline 1963 & 172.587 & 9.581 & $\mathbf{1 8 2 . 1 6 8}$ \\
\hline 1964 & 194.600 & 12.454 & $\mathbf{2 0 7 . 0 5 4}$ \\
\hline 1965 & 207.650 & 15.253 & $\mathbf{2 2 2 . 9 0 3}$ \\
\hline 1966 & 220.522 & 17.548 & $\mathbf{2 3 8 . 0 7 0}$ \\
\hline 1967 & 225.977 & 20.771 & $\mathbf{2 4 6 . 7 4 8}$ \\
\hline 1968 & 191.480 & 26.819 & $\mathbf{2 1 8 . 2 9 9}$ \\
\hline 1969 & 200.504 & 33.052 & $\mathbf{2 3 3 . 5 5 6}$ \\
\hline 1970 & 220.684 & 40.658 & $\mathbf{2 6 1 . 3 4 2}$ \\
\hline 1971 & 244.900 & 43.551 & $\mathbf{2 8 8 . 4 5 1}$ \\
\hline 1972 & 280.007 & 47.734 & $\mathbf{3 2 7 . 7 4 1}$ \\
\hline 1973 & 351.852 & 49.482 & $\mathbf{4 0 1 . 3 3 4}$ \\
\hline 1974 & 430.702 & 49.833 & $\mathbf{4 8 0 . 5 3 5}$ \\
\hline 1975 & 431.454 & 56.177 & $\mathbf{4 8 7 . 6 3 1}$ \\
\hline 1976 & 430.024 & 57.823 & $\mathbf{4 8 7 . 8 4 7}$ \\
\hline 1977 & 359.998 & 60.552 & $\mathbf{4 2 0 . 5 5 0}$ \\
\hline 1978 & 329.007 & 65.670 & $\mathbf{3 9 4 . 6 7 7}$ \\
\hline 1979 & 321.819 & 69.908 & $\mathbf{3 9 1 . 7 2 7}$ \\
\hline 1980 & 315.931 & 71.334 & $\mathbf{3 8 7 . 2 6 5}$ \\
\hline 196 & 596 & 2008 \\
\hline
\end{tabular}

Fuente: Secretaría de Políticas Universitarias, 2008

En efecto, estos sectores pensaban que el crecimiento de la matrícula debía acompañarse con un cambio en la oferta universitaria, orientado en dos sentidos: la división de las universidades existentes en conglomerados más o menos uniformes y la creación de nuevas universidades. Es decir, ante la expansión numérica de los estudiantes universitarios se proponía descentralizar el sistema de educación superior y extender el número de instituciones que lo integraban. Como representante de los sectores liberales católicos dentro de la UBA, el proyecto del entonces decano de la Facultad de Farmacia y Bioquímica se inscribió en una lectura particular de la experiencia renovadora de la universidad pública entre 1955 y 1966. Su diagnóstico alertaba sobre la incompatibilidad entre la universidad de 
masas y la universidad científica. De tal forma, la idea de fundar nuevas universidades también tenía como propósito crear dos circuitos universitarios: uno de investigación de elite, y otro profesionalista que atendiera a las demandas crecientes por educación superior.

Frente a lo que consideraba el "[...] trascendente y distorsionante peso estructural de la Universidad de Buenos Aires" (Taquini, 1984), Taquini recomendó la división de esa institución por áreas geográficas que posteriormente podrían independizarse: UBA II (en la Ciudad Universitaria de Núñez), UBA III (en el área que rodea a Plaza Houssay) y UBA IV (en lo predios de la Facultad de Agronomía y Veterinaria). ${ }^{16}$ El Rectorado, junto con las restantes dependencias, conformaría la UBA I (Ibídem).

En 1968, el entonces rector de la universidad Raúl Luis Devoto -nombrado en el cargo por un decreto del Poder Ejecutivo Nacional- preparó un "Plan de Transformación de la Universidad de Buenos Aires", que incluía parcialmente el proyecto de división de la universidad junto con otras medidas como la departamentalización, un nuevo sistema de ingreso por fuera de las Facultades y el cierre de la ciudad universitaria. El conjunto de las medidas suscitaron la oposición de los sectores estudiantiles, de numerosos docentes y de los decanos de las Facultades de Filosofía y Letras, Odontología y Derecho, quienes presentaron su renuncia al rectorado en señal de protesta por no haber sido consultados acerca de la implantación de dichos cambios.

En ese marco, el rector Devoto se vio obligado a renunciar a su cargo y, junto con el fracaso de su gestión, el proyecto de división de la universidad quedó de lado. ${ }^{17}$ A partir de allí, las demandas de Taquini y su grupo de expertos se concentrarían en torno a la creación de nuevas universidades, diferenciadas de las tradicionales. De ese modo, la alta concentración estudiantil en la Universidad de Buenos Aires quedaría sin resolverse y permanecería como una preocupación latente del gobierno y los sectores dominantes debido a su posible impacto político y social. En tal sentido, el Plan de Nuevas Universidades propiciaba:

"Nuevas universidades, independientes de los centros actuales de educación superior, libres de sus problemas, de orientación renovadora; no atadas a los intereses del continuismo ni del paternalismo académico de las actuales universidades [...]" (Taquini, 1972: XIII).

Originariamente, se proyectó la fundación de cinco casas de estudio, de acuerdo a dos criterios determinantes: la concentración poblacional y la necesidad de impulsar el crecimiento zonal como resultado de la instalación de centros de enseñanza superior y de investigación en dichas áreas. Siguiendo el primero, se emplazaron dos instituciones en el conurbano bonaerense: una en el oeste (Luján) y la segunda en el sur, en el trayecto entre Buenos Aires y La Plata (Quilmes). Tomando en consideración el segundo propósito, se pretendía crear una universidad en la Patagonia, mientras que la creación de una universidad en el sur de la provincia de Córdoba (Río Cuarto) y de otra localizada en la zona norte de Buenos Aires, próxima a Zárate, pretendía conjugar ambos objetivos. 
El modelo de universidad propuesto por estos sectores, influenciado por el modelo de las research universities norteamericanas, contemplaba la departamentalización de la universidad, en oposición al sistema de Facultades y cátedras; la oferta de carreras no tradicionales; el aporte al conocimiento científico-tecnológico; la creación de campus universitarios (o ciudades universitarias); la inserción de la universidad en el aparato productivo del medio, la región o la comunidad; y la expansión matricular dentro de un tamaño óptimo estimado entre los $15.000 \mathrm{y}$ 25.000 alumnos.

Con respecto a la estructura universitaria, la propuesta planteaba crear universidades próximas a centros urbanos de mediana dimensión, pero también aisladas en cierta medida de éstos. En efecto, el plan rechazaba la existencia de Facultades dispersas o lejanas a un centro urbano pero también desaconsejaba, en los casos en que la población estudiantil excediese los 15.000 alumnos, la ubicación de una ciudad universitaria o campus en el interior de un complejo urbano.

Con relación al vínculo con el medio, se insistió en la necesidad de crear nuevas universidades con el propósito de evitar la emigración interna, al situar a las nuevas instituciones en áreas que por su densidad poblacional lo requirieran, y de contribuir al aporte científico, cultural y técnico de las mismas. Para ello, se fomentaba la creación de carreras novedosas, distintas en lo posible de las tradicionales, y el otorgamiento de títulos intermedios. El plan asociaba el concepto de universidad regional a la idea de una institución que debía responder a las necesidades derivadas del desarrollo regional.

A pesar de este ascenso de la problemática universitaria en la esfera estatal, la cuestión de la creación de nuevas universidades no logró mayores apoyos. Si bien en 1968 se aprobó la creación de la Universidad Nacional de Rosario (UNR) en el marco de la Ley Orgánica de Universidades Nacionales $\mathrm{N}^{\circ} 17.245$, la propuesta pareció responder más a sus antecedentes históricos (varios proyectos de ley de creación de la Universidad Nacional de Rosario, anteriores a la fundación de la UNL) y a una evidente superposición de estructuras entre las dos sedes de la UNL (Rosario y Santa Fe/Paraná) que a un intento efectivo de concretar del plan de nuevas universidades. Tal como fue desarrollado anteriormente, el régimen autoritario de Onganía concentró sus fuerzas en la regulación de las instituciones y los sujetos universitarios y en el redimensionamiento de las universidades a través de la promoción de mecanismos selectivos de admisión. Así, la posibilidad de crear nuevas universidades nacionales fue interpretada como una amenaza para la conquista de esos objetivos y no como un medio para encauzar el proceso de participación política.

Hacia 1968, Taquini y su grupo de expertos reiteraron su voluntad de crear nuevas universidades presionando sobre el gobierno nacional. Abocados a la creación de la Universidad Nacional de Río Cuarto, se entrevistaron con distintos funcionarios para captar sus adhesiones. Antes de ser depuesto, Onganía se comprometió públicamente a estudiar la posibilidad de creación de la Universidad Nacional de Río Cuarto, pero su fundación se demoró como consecuencia de los distintos estudios de factibilidad. Dichos estudios fueron utilizados desde las agencias es- 
tatales como recursos dilatorios ante el aumento de pedidos para fundar nuevas instituciones.

La cuestión universitaria alcanzaría una mayor jerarquía institucional durante la presidencia de facto de Levingston y la conducción ministerial de José Luís Cantini. En efecto, se conformaron cinco subsecretarías: Técnica, de Supervisión Escolar, Universitaria, Económica-financiera y de Cultura. A su vez, al ámbito de la Subsecretaría Universitaria se incorporó la Dirección Nacional de Altos Estudios, junto con tres departamentos: supervisión de universidades privadas, supervisión de universidades provinciales y títulos.

Si bien en los primeros meses de su breve gobierno la política prioritaria en materia universitaria trató de implementar la ley de universidades nacionales $\mathrm{N}^{\circ} 17.245$ recientemente sancionada, frente a los hechos conocidos como Viborazo Levingston advirtió la posibilidad de contener al movimiento estudiantil creando nuevas universidades. ${ }^{18}$ En una audiencia con representantes de la Comisión Pro-Universidad de Río Cuarto, manifestó su preocupación por "el problema obrero en la ciudad de Córdoba (...) que insumía grandes energías al gobierno" (Martorelli, 1991: 152) y destacó la necesidad de una descentralización universitaria “[...] que evite la aglomeración estudiantil, que se traduce, con frecuencia, en hechos que suelen afectar la tranquilidad pública" (Ibídem). Antes de la finalización de su mandato, Levingston encargó al CONADE la inclusión de la propuesta de creación de nuevas universidades en el llamado "Plan Nacional de Desarrollo y Seguridad 1971-1975”. En suma, la idea de creación de nuevas universidades ingresó finalmente a la agenda de controversia pública; en cuanto política pública a ser desarrollada en los siguientes cuatro años, marcó un verdadero cambio de rumbo para el sector universitario. El Plan Nacional fue aprobado finalmente por el decreto-ley $\mathrm{N}^{\circ} 19.039$ con la firma del nuevo presidente de facto, Agustín Lanusse.

Los proyectos de los técnicos ministeriales tomaron la mayor parte de los lineamientos del Plan de Nuevas Universidades del grupo de Taquini aunque ampliaron los alcances de la propuesta: el documento sostuvo que en 1972 se procedería al ordenamiento geográfico de la Universidad Nacional de Cuyo, lo cual implicaba la creación de instituciones autónomas en San Juan y San Luis a partir de las sedes de la primera. Luego, se priorizó la creación de la Universidad Nacional de Río Cuarto y la de dos instituciones al oeste y al sur del conurbano bonaerense: Luján y Lomas de Zamora. ${ }^{19}$ De tal forma, en el proceso mismo de elaboración de la política de expansión institucional y ante ausencia de partidos políticos que pudieran procesar las distintas demandas sociales de educación superior, los especialistas en temas universitarios oficiaron como mediadores de los intereses de ciertos grupos regionales y locales que intentaban instalar sus demandas en la esfera estatal.

Siguiendo esa línea, si bien el Plan de Nuevas Universidades se presentó como un informe técnico-descriptivo de la "realidad" universitaria del país, su contenido fue objeto de fuertes luchas políticas y sociales y una apuesta más -entre otrasacerca de cómo debía ser la universidad. En tal sentido, fue exitoso en cuanto a la instalación en la agenda de controversia pública de una representación de las 
universidades nacionales tradicionales en términos de instituciones "sobredimensionadas", "congestionadas" e incluso "monstruosas". Desde esta perspectiva, el conflicto universitario, asociado a problemas de funcionamiento y rendimiento, se presentó como el resultado del crecimiento de la población estudiantil; se pretendió resolverlos mediante la distribución espacial en campus, dotándolas de una estructura departamental. Esas representaciones universitarias adquirieron un efecto naturalizador, que tendió a velar el carácter construido de las realidades descriptas y también ocultó su pretensión performativa.

De tal forma, las figuras del intelectual y del experto conformaron durante este período un espacio de intersección productiva (Neiburg y Plotkin, 2004) en la esfera estatal donde circularon individuos, ideas, modelos educativos y desde donde se produjo conocimiento social sobre las poblaciones que se pretendía gobernar. Por otra parte, el ascenso de la figura del especialista en educación, a través de la consagración de los valores de modernización e innovación, supuso una estrategia de intervención común -aunque de ninguna manera libre de contradicciones internas y tensiones- desplegada por los grupos católicos y liberales modernizadores (Suansábar, 2004) quienes actuaron como parte interesada en las luchas en torno de la definición del papel del Estado y de la definición de sus políticas.

\section{Las ideas en la política: fragmentación, dispersión y conciliación}

La asunción de Lanusse como presidente, significó un viraje en la política de la autodenominada Revolución Argentina, al proponer en nombre de las Fuerzas Armadas un "Gran Acuerdo Nacional (GAN)" entre militares y civiles que garantizara una salida electoral para canalizar el descontento y la radicalización social. Al incluir al peronismo, la reapertura de la actividad política pretendía aislar todas las variantes del "partido armado" y desactivar a la oposición civil (Altamirano, 2001: 92) como parte de la percepción gubernamental de la existencia de una amenaza generalizada por el carácter nacional y socialmente heterogéneo de la protesta.

El $1^{\circ}$ de mayo de 1971, desde las escalinatas del Palacio Municipal de la ciudad de Río Cuarto y ante una multitud entusiasmada, el presidente de facto inauguró la primera de las nuevas universidades proyectada en el Plan Nacional de Desarrollo y Seguridad (1971-1975). Con posterioridad a ese discurso, Lanusse dirigió un mensaje al país donde intentó fijar algunas de las condiciones del acuerdo nacional. En líneas generales, el GAN planteaba una alianza entre amigos y adversarios para enfrentar a los nuevos enemigos. Los enemigos del pasado -los peronistas- pasaron a conformar la categoría de adversarios, mientras que los grupos más radicalizados -en lucha contra el régimen autoritario y el modelo de acumulación capitalista existente- constituyeron el principal foco de preocupación y amenaza para el gobierno.

Pocos meses antes, un comunicado del gobierno difundido en la prensa nacional, había advertido sobre la cooptación de la juventud y el movimiento obrero por "grupos ideológicamente extranjeros", cuyos intereses "antiargentinos" intentaban cambiar el "claro sentido nacional" de los trabajadores y "apartar de la disciplina del estudio a nuestra juventud universitaria". ${ }^{20}$ No resulta extraño, entonces, que 
en el discurso en Río Cuarto el presidente realizara una convocatoria especial a "la juventud argentina", a quien invitaba a conformar el proceso de apertura política a través de la consolidación de "[...] partidos modernos y verdaderamente representativos"; de esa manera, pensaba brindarles "[...] los canales adecuados para encauzar sus ideas e inquietudes, dentro del marco de la constitución y de la ley". ${ }^{21}$ Asimismo, efectuaba un llamamiento "[...] a sumarse al franco debate [...] y comprometerse en la gran tarea de imaginar y construir la Argentina del futuro". ${ }^{22}$

El gobierno proponía reencauzar la lucha estudiantil por los rieles menos peligrosos de la democracia electoral. Como contrapartida, ofrecía una nueva universidad y prometía, siguiendo los lineamientos del Plan Nacional, que "[...] a esta casa de altos estudios seguirá la Universidad Nacional del Comahue y también posibilitaremos la instalación de una Universidad en Concepción del Uruguay" (Ibídem). Tres meses después, en ocasión de la apertura de la Universidad Provincial de la Rioja, el nuevo Ministro de Cultura y Educación, se refería a la "cuestión estudiantil" afirmando que "éste es el triunfo de ustedes, de la juventud riojana que, como un ejemplo para todo el país, no ha elegido el camino del incendio ni de la destrucción, sino el camino positivo". ${ }^{23}$

Como parte de una estrategia tendiente a fragmentar al movimiento estudiantil y cooptar apoyos de los sectores menos radicalizados, el régimen autoritario se refería a éstos en términos de "esperanza y promesa". En el marco de una iniciativa estatal más amplia destinada a deteriorar los vínculos entre los distintos sectores sociales activados y las vanguardias revolucionarias (Tortti, 1999: 208), las nuevas universidades nacionales fueron identificadas como "canales adecuados de participación" ${ }^{24}$ para aquellos jóvenes, mientras que la posibilidad de reducir las incertidumbres derivadas de la alta conflictividad social promovió el acercamiento a las propuestas de los grupos técnicos. Por otra parte, los hechos de la denominada "Masacre de Trelew", revelarían la brutalidad con la que el régimen autoritario actuaba con aquellos a quienes consideraban sus enemigos. ${ }^{25}$

En el plano burocrático-estatal de la esfera educativa tuvo lugar un desplazamiento de los expertos nacionalistas-católicos y liberales modernizadores, como resultado de la nueva conducción encabezada por Gustavo Malek, rector de la Universidad Nacional del Sur. ${ }^{26}$ Como parte de los esfuerzos tendientes a devolver legitimidad a las acciones del Estado, la arena educativa se expandió sin la ampliación correspondiente de las capacidades para llevarlas a cabo. ${ }^{27}$ En tanto reflejo del conflicto social en la esfera estatal, se superpusieron funciones y surgieron "sectores multipresentes" con denominaciones profusas o vagas. Así, la estructura del ministerio de Cultura y Educación en el período 1968-69 en comparación con la de 1972-73, evidenció un incremento del $40 \%$ del número de agencias públicas (Ferrauti y Lentini, 1979: 26). Ese crecimiento estuvo centrado en las funciones de gestión y/o control del planeamiento. En tal sentido, la creación de dos Direcciones Nacionales, seis Direcciones Generales y doce sectores, produjo un alto grado de dispersión del planeamiento, la programación y el control de la gestión.

Ante la decisión política de aumentar el número de universidades nacionales, se constituyeron otras dos comisiones para tratar la ampliación del sistema 
universitario y se emitió un documento que establecía ciertas pautas para la presentación de proyectos de creación de universidades nacionales. En una primera etapa, la normativa solicitó información mínima para estudiar la viabilidad del proyecto, mientras en una segunda instancia se requirieron datos acerca de la estructura institucional de la futura universidad. Se advertía la conveniencia de formar una comisión de trabajo con apoyatura técnica. Así, los especialistas en educación superior comenzaron a circular por las comisiones pro-fundacionales para aportar su saber, al que se consideraba regido por criterios objetivos y neutrales. La solicitud de extensos cuerpos estadísticos y documentales que pudieran avalar la creación de nuevas universidades, intentó introducir cierta racionalidad de carácter técnico en el proceso de implementación de la política. En tal sentido, se establecía una relación de dependencia mutua entre " [...] la elaboración e implementación de políticas estatales por parte de los agentes de la administración y la producción de conocimiento sobre las poblaciones" (De L'Estoile et al., 2002: 9) donde se asentarían las nuevas universidades, y a las cuales se aspiraba gobernar.

\section{Cuadro II- Crecimiento de Universidades Públicas y Privadas en el período 1955-1973}

\begin{tabular}{|c|c|c|c|c|c|}
\hline $\begin{array}{c}\text { Instituciones } \\
\text { Universitarias }\end{array}$ & Hasta 1957 & \multicolumn{2}{|c|}{$1958-1970$} & \multicolumn{2}{|c|}{$1971-1973$} \\
\hline Ámbito & Público & Público & Privado & Público & Privado \\
\hline Crecimiento & - & +2 & +19 & +13 & +1 \\
\hline Total & 8 & 10 & 19 & 23 & 20 \\
\hline
\end{tabular}

Fuente: Elaboración propia en base a datos del Anuario 2005 de Estadísticas Universitarias, de la Secretaría de Políticas Universitarias

Esos requerimientos de información desembocaron en la adquisición de una posición privilegiada por parte de los expertos al situarlos en el centro de las luchas por la definición del papel del propio Estado. La centralidad de esas figuras en la elaboración de estudios de factibilidad y posterior evaluación en el ámbito ministerial, ocultaba la naturaleza también política de los actores involucrados en el proceso. En este período, el pasaje de distintas personalidades desde la esfera ministerial hacia las comisiones pro-universidad, y desde éstas hacia otros ámbitos políticos fueron un hecho recurrente. ${ }^{28}$

De tal forma, el Estado autoritario terminó de conformar, aunque frágilmente y con no pocas tensiones, un campo de políticas universitarias, resultado de la expansión "[...] tanto de las instituciones de educación superior como del aparato gubernamental [...]" (Cox, 1993) que pretendió regularla (ver el cuadro II). Por su parte, los estudios de factibilidad que pretendieron avalar el surgimiento de las nuevas casas de estudio modificaron la forma y el contenido de los proyectos universitarios. La circulación de esos informes a través de las fronteras estatales de la 
mano de especialistas universitarios, produjo importantes cambios en el proceso mismo de elaboración de las políticas.

No obstante, con la creación de nuevas universidades a principios de los años 70 , el régimen autoritario introdujo una dinámica institucional específica en el rumbo de las políticas universitarias. En primer lugar, la organización burocrática, en tanto racionalidad administrativa, y la coordinación tecnocrática requerida por la complejidad de los sistemas de educación superior, fueron muy débiles. Las estructuras organizativas del sector educativo generaron una fuerte dispersión en la toma de decisiones, lo cual amplió las posibilidades de especialistas y consultores universitarios quienes, a través de contactos y redes de relaciones personales en el Estado, torcieron -en muchos casos- a favor de sus propios intereses la habilitación de nuevas instituciones. De tal forma, a la difícil relación de competencia entre la burocracia y la tecnocracia en las fronteras estatales, se sumaron lógicas predatorias que complejizaron la implementación de la política de expansión universitaria.

Como oportunamente señalaron varios autores (O'Donnell, 1979 y 1982; De Riz, 2000; Altamirano, 2001), la cuestión del tiempo fue un problema crucial para el Estado y marcó las políticas del período. En particular, la creencia en un inminente desmoronamiento, o simplemente en un colapso del régimen autoritario, encuadró la expansión universitaria en un tiempo urgido por la coyuntura, lo que contribuyó a acelerar la creación de algunas casas de estudio. Esas creencias y racionalidades de la política quedaron inscriptas como marcas de origen en las nuevas instituciones. En efecto, durante el período fundacional, las nuevas universidades exhibieron una gran vulnerabilidad, la que puede interpretarse como la manifestación de un fenómeno más amplio relacionado con la propia debilidad del Estado, y más precisamente, de sus estructuras en el contexto de su surgimiento (Sigal, 2002: 49).

\section{Consideraciones finales}

Al comenzar este trabajo se propuso analizar los diversos circuitos de producción de la "idea" de fundación de nuevas universidades nacionales, mostrando cómo se constituyó particularmente en el aparato burocrático-estatal. En el camino recorrido, se procuró identificar los distintos posicionamientos que se originaron frente a la propuesta, así como las razones que llevaron al Estado a adoptar decisiones en esa dirección.

Sintetizando, en los años 50 y 60 la preocupación por el número de universidades surgió junto con la cuestión del desarrollo, asignándole a la universidad un rol privilegiado como motor del proceso. De ese modo, la expansión universitaria se vinculó estrechamente con el ritmo de la modernización económica y social en curso, lo que dio lugar a un debate cualitativo sobre la misión de dichas instituciones.

Sin embargo, los movimientos modernizadores no pudieron completarse debido a las discontinuidades político-institucionales y a la resistencia de algunos actores universitarios. La dinámica de crecimiento cobró la forma de un agregado de capas originadas en tradiciones divergentes, lo que generó un entramado he- 
terogéneo que se expresó en la universidad primero, y en el sistema nacional de educación superior después (Naishtat et al., 2005).

En ese marco, el Plan de Nuevas Universidades, elaborado a fines de la década de 1960, combinó criterios modernizadores y elititas, que reprodujeron los conflictos propios del período anterior. Sus éxitos consistieron en recuperar algunos proyectos que habían quedado inacabados, en cautivar el interés de las poblaciones rezagadas por falta de una oferta de educación superior, y en imaginar nuevas realidades derivadas del desarrollo científico y humano que podían proveer las universidades. Sobre este último punto, cabe destacar que si bien la propuesta formuló un modelo innovador, también concibió a las nuevas universidades nacionales como centros de formación de recursos humanos y alternativas para el descongestionamiento de la demanda profesional de las universidades metropolitanas.

En el contexto del proyecto autoritario, las ideas de seguridad y modernización cambiaron el eje analítico: las universidades nacionales de más larga tradición, definidas como "motores del desarrollo", pasaron a ser "monstruos sobredimensionados". En este nivel, las medidas gubernamentales buscaron desmontar y detener el crecimiento de la matrícula y su concentración en los centros urbanos, de modo que la cuestión universitaria parecía consistir en un problema puramente cuantitativo. Pero el criterio de la cantidad ocultaba la voluntad de contrarrestar al activismo estudiantil de dichas instituciones.

En el gobierno de facto de Lanusse, el tránsito desde el Plan de Nuevas Universidades a la política de expansión universitaria, implicó una estrategia destinada a fragmentar al movimiento estudiantil y cooptar apoyos de los sectores menos radicalizados. Así, las futuras universidades se imaginaron como "canales adecuados de participación" para los jóvenes. La convocatoria a presentar nuevos proyectos universitarios produjo una ampliación en el número de las futuras instituciones en comparación con las que habían sido proyectadas originariamente. El itinerario del plan a la política estuvo mediado por la participación de expertos en temas universitarios quienes, frente a la supresión del juego democrático-liberal, procesaron las demandas crecientes de fundación de instituciones de educación superior. A su vez, las propuestas se fundamentaron a través de la elaboración de distintos estudios de factibilidad, beneficiados por el instrumental teórico de las ciencias sociales.

De allí que el análisis realizado trasluce la existencia simultánea de dos tendencias contrapuestas: por un lado, las propuestas fundacionales se ligaron a las demandas del entorno, entendida la región como un ámbito sobre el que podían aplicarse los conocimientos científicos en pos del crecimiento y el desarrollo de las poblaciones locales; por otro, como toda creación institucional, los proyectos reflejaron las disputas políticas de la época, las relaciones de fuerza entre grupos de expertos y fracciones de la burocracia estatal, y las estrategias diseñadas por el gobierno para conducir y controlar la situación general, incluida la efervescencia social del período.

Con ello, la complejidad del proceso que culminó con la creación de universidades nacionales puso de manifiesto no sólo la existencia de tendencias académi- 
cas discordantes, sino también de ideologías políticas diversas. Puede decirse que entre ambas -y a lo largo de todo el período- se verificó una intrincada relación cuyas repercusiones bosquejaron un campo atravesado por contradicciones, paradojas y confusiones: saberes técnicos enfocados a la especificidad de las misiones $\mathrm{y}$ funciones de las universidades regionales, junto con decisiones políticas que excedían largamente dichas dimensiones. ${ }^{2}$

\section{Referencias}

1. Las universidades nacionales pasaron de diez a veintitrés. Sin embargo, sólo cinco fueron realmente nuevas: Río Cuarto, Lomas de Zamora, Luján, Santiago del Estero y Catamarca. Otras cinco instituciones se originaron por subdivisiones: Salta, San Juan, San Luis, Entre Ríos y Misiones. Las tres restantes fueron nacionalizaciones de universidades anteriores provinciales o privadas: Comahue, La Pampa y Jujuy.

2. Posteriormente, se nacionalizaron las universidades del Centro de la Pcia. de Bs. As. (1974) y Mar del Plata (1975) y se creó la Universidad de la Patagonia (1980).

3. Al decir de Sigal (2002: 21) así lo testimonian la Reforma Universitaria de 1918 y las perturbaciones producidas tanto por los golpes de Estado de 1930, 1943, 1955, 1966 y 1976 como las sucesiones electorales. Por tanto, la historia política argentina provee una periodización posible de las historia de las universidades.

4. En particular, nos referimos al escenario político-institucional pos-Cordobazo, en el que se extendieron los procesos de activación social y se profundizaron las manifestaciones de radicalización política.

5. Así lo documentan, entre otros, los trabajos fundacionales de Terán (1991) y Sigal (1991).

6. La autodenominada Revolución Argentina, producto del golpe militar de 1966, se extendió hasta 1973.

7. Ley $17.245 / 67$.

8. Conviene recordar que de acuerdo al célebre estudio del Centro de Investigaciones Sociales del Instituto Di Tella conducido por Enrique Oteiza y Marta Slemenson en 1970, también en la UBA, la reacción de los docentes frente a la intervención de 1966 adquirió características disímiles en las distintas Facultades. Así, de las 1.315 renuncias docentes sucedidas después de la intervención, el $51.35 \%$ correspondió a la Facultad de Ciencias Exactas y el $41.42 \%$ a Filosofía y Letras. En Medicina sólo renunció un $2.59 \%$ de los profesores y en Odontología directamente no las hubo. El departamento de Física de Ciencias Exactas quedó prácticamente sin docentes al igual que la carrera de Psicología. En Arquitectura también dimitieron 268 docentes. En: Análisis, № 460, 6 al 12 de enero de 1970.

9. Algunos de los itinerarios de esos académicos fueron: el Instituto Campomar o la Fundación Bariloche para un pequeño grupo de Exactas y en el caso de las ciencias sociales, el Instituto Di Tella, IDES, CLACSO, entre otros. En el plano local, algunos profesores de las Universidades de Buenos Aires y La Plata circularon por el Instituto Universitario de Olavarría, y las universidades provinciales de Tandil y Mar del Plata mientras que, a nivel regional, Chile, Venezuela, Uruguay y México fueron los principales destinos del éxodo de científicos y profesionales.

10. Dicha agencia estatal fue conducida primero por Carlos Gelly y Obes y luego, por José Mariano Astigueta, quien estaría a cargo del controvertido "Anteproyecto de Reforma del Sistema Educativo Argentino" mediante el cual se produjo una transformación de la estructura del sistema al introducir un nivel intermedio entre la escuela primaria y secundaria. Sobre esa reforma y su impacto, puede consultarse el trabajo de Braslavsky (1982).

11. Entre 1958 y 1971 se crearon veintiuna universidades privadas. Conviene señalar que la normativa implementada en este período para regular al sector estuvo en vigencia hasta la sanción de la Ley de Educación Superior (24.521) de 1995.

12. Entendemos aquí a la noción de tecnocracia como "[...] el poder, otorgado a los especialistas, de tomar decisiones en función fundamentalmente de su competencia técnica" (Loureiro, 1997: 128). En 
un marco autoritario como el de la Revolución Argentina, al no estar las decisiones de los especialistas sometidas a control o a la posibilidad de ser cuestionadas por la población, el término adquiere un sentido estrictamente antidemocrático.

13. Mignone fue uno de los exponentes más interesantes de ese recorrido: desde muy joven formó parte de esa nueva generación dentro del catolicismo identificada fuertemente con el programa del humanismo cristiano; se recibió de abogado en la UBA y, entre 1949 y 1952, con veintiséis años de edad, fue Director General de Enseñanza de la Provincia de Buenos Aires. A mediados de los 60, trabajó como especialista en educación en el Departamento de Cooperación Técnica de la Organización de Estados Americanos (OEA) en Washington y en el Banco Interamericano de Desarrollo. A su regreso al país, a fines de 1967, dirigió el sector Educación Superior del Consejo Nacional de Desarrollo (CONADE), y entre junio de 1969 y mayo de 1971, fue designado al frente de la Subsecretaría de Educación, y luego, de la Subsecretaría Técnica del Ministerio de Cultura y Educación. En junio de 1973 fue nombrado rector de la Universidad Nacional de Luján por el gobierno peronista de Héctor J. Cámpora. En 1977, tras el secuestro de su hija Mónica, hasta hoy desaparecida, fue uno de los primeros en denunciar al régimen militar por la aplicación sistemática de torturas. Un año después, fundó el Centro de Estudios Legales y Sociales (CELS) y participó del Instituto Interamericano de Derechos Humanos. En 1996 fue nombrado miembro de la Academia Nacional de Educación y era el presidente de la Comisión Nacional de Evaluación y Acreditación Universitaria al momento de su muerte en 1998.

14. Esas preocupaciones se enmarcaron en el clima de efervescencia revolucionaria de la época, cuando en 1968 las ciudades Berkeley, Tokio, Roma, Berlín, París, Varsovia y México D.F. fueron los epicentros de diversos estallidos de rebelión juvenil.

15. La noción de agenda de controversia pública refiere a aquellos temas que no cumplen con un estándar evaluativo y, por lo tanto, son reconocidos como problemas y ponen en marcha determinados cursos de acción gubernamental (ver, entre otros, Cox, 1993).

16. Corresponde señalar que durante el rectorado de la UBA de Hilario Fernández Long (1964-1966) se delineó un primer proyecto de descentralización de la institución.

17. En el período de la transición democrática, el rector normalizador de la UBA, Francisco Delich, impulsaría un proyecto de descentralización de esa institución, el cual reformulaba la idea de división de esa casa de estudios en distintas unidades académicas. De todas formas, la propuesta tampoco logró implementarse. Al respecto puede consultarse el trabajo de Rovelli (2008).

18. A fines de febrero de 1971 Levingston designó como gobernador de Córdoba a Camilo J. Uriburu (sobrino del general golpista del 30) quien anunció su decisión de cortar la cabeza de la víbora de la subversión. El 12 de marzo los sindicatos cordobeses dispusieron una huelga general que contó con el apoyo de los estudiantes. Actuó la policía, pero el 15 la violencia volvió a estallar. Hubo enfrentamientos y saqueos, aunque la fuerza pública logró dominar la situación. Desde entonces, los comandantes de las tres fuerzas consideraron que el tiempo de la denominada Revolución Argentina había expirado. Levingston fue destituido y la Junta Militar asumió el poder, designando como presidente a Lanusse.

19. Lomas de Zamora logró desplazar el proyecto universitario originario situado en Quilmes. Fue la propia comisión pro-universidad de Lomas de Zamora la que convenció a Taquini de emplazar la nueva universidad en el predio de Santa Catalina en Llavallol, perteneciente al instituto Fitotécnico de la Universidad Nacional de La Plata.

20. "Grupos ideológicamente extranjeros", en La Nación, 15/3/71.

21. Por otra parte, conviene recordar que unos días antes de ese discurso, el 29 de abril, un grupo armado había asesinado al teniente Marcos César Assúa, lo que fue tomado por el propio Lanusse como una provocación política a la que debía responder en su alocución (Lanusse, 1977: 234).

22. "General Alejandro A. Lanusse. Mensaje al país desde Río Cuarto, 1ro. de mayo de 1971", en Clarin 2/05/71.

23. Universidad Nacional de la Rioja, Historia de la Universidad Nacional de la Rioja [en línea], obtenida el 13 de marzo de 2006, disponible en: http://www.unlar.edu.ar/web/historia.htm

24. "General Alejandro A. Lanusse. Mensaje al país desde Río Cuarto, 1ro. de mayo de 1971", en Clarin 2/05/71. 
25. E1 22 de agosto de 1972 en la base naval Almirante Zar, fueron asesinados dieciséis presos políticos que habían sido trasladados allí, seis días antes, luego de que se efectivizara una acción conjunta de las organizaciones Fuerzas Armadas Revolucionarias (FAR), Ejército Revolucionario del Pueblo (ERP) y Montoneros. Ello permitió la evasión de seis jefes guerrilleros recluidos en la cárcel de Trelew, quienes tras abordar un avión civil previamente secuestrado por un grupo comando, lograron refugiarse en Chile, gobernado por el socialista Salvador Allende.

26. Lanusse le ofreció la conducción del Ministerio de Cultura y Educación a Antonio Salonia, militante de la Unión Cívica Radical, funcionario del área educativa durante el gobierno de Frondizi y la gestión de Pérez Guilhou. Frente a su rechazo, asumió el cargo el rector de la Universidad Nacional del Sur. El testimonio fue recogido en la entrevista realizada al Prof. Salonia en junio de 2007.

27. De acuerdo a Sikkink (1991: 11), "las capacidades estatales incluyen a las habilidades administrativas y coercitivas del aparato estatal para implementar metas oficiales. La capacidad estatal es un concepto relacional: el estado es fuerte o capaz en relación con las tareas que se fija para sí mismo o que son finadas para él".

28. A modo de ejemplo, vale el caso de Mignone, quien en 1971 renunció a su cargo de Subsecretario de Educación, después de la asunción de Lanusse a la presidencia. Ese mismo año, comenzó a colaborar como consultor en la elaboración del estudio de factibilidad de la Comisión Pro-Universidad Nacional de Luján. En el caso de la Universidad Nacional de Lomas de Zamora, el Brigadier (RE) Miguel Moragues, integrante de la comisión Pro-Universidad, fue nombrado gobernador de Buenos Aires, mientras el presidente de esa comisión, Enrique Roig, fue designado ministro de gobierno (Estudio de factibilidad, Tomo I, 1972).

\section{Bibliografía}

C. ALTAMIRANO (2001), Bajo el signo de las masas (1943-1973), Buenos Aires, Ariel Historia. W. ANSALDI (2004), "Matriuskas de terror. Algunos elementos para analizar la dictadura argentina dentro de las dictaduras del Cono Sur", en A. PUCCIARELLI (comp.) La dictadura militar y el origen del liberalismo corporativo, Buenos Aires, Siglo Veintiuno Argentina Editores.

M. L. BERTONI y D. J. CANO (1990), "La educación superior argentina en los últimos veinte años: tendencias y políticas", en Revista Propuesta Educativa, Buenos Aires, FLACSO, pp. 11-24.

C. BRASLAVSKY (1980), "La educación argentina (1955-1980)", Primera Historia Integral, Buenos Aires, CEAL.

P. BUCHBINDER (2005), Historia de las Universidades Argentinas, Buenos Aires, Editorial Sudamericana.

M. CAVAROZZI (1983), Autoritarismo y democracia (1955-1983), Buenos Aires, CEAL.

A. CHIROLEU (2004), "La modernización universitaria en la agenda de gobierno argentino", en Fundamentos en humanidades. Universidad de San Luis, Año V, Nro. I, San Luis.

C. COX (1993), "Políticas de educación superior, categorías para su análisis", en H. COURARD (comp.), Políticas comparadas de educación superior en América Latina, Santiago de Chile, FLACSO.

L. DE RIZ (2000), La politica en suspenso, Buenos Aires, Paidós.

P. EVANS, D. RUESCHEMEYER, T. SKOCPOL (editors) (1985), Bringing the State Back in, London, Cambridge University Press.

P. KROTSCH (2001), Educación Superior y Reforma Comparada, Buenos Aires, Universidad Nacional de Quilmes Editorial.

B. L'ESTOILE, F. NEIBURG, y L. SIGAUD (2002), “Antropologia, impérios e estados nacionais: uma abordagem comparativa”, en B. L'ESTOILE, F. NEIBURG y L. SIGAUD (orgs.), Antropologia, Império e Estados Nacionais, Rio de Janeriro, FAPERJ, Relume-Dumará.

M. R. LOUREIRO (1997), “Tecnocracia y política en el Brasil de hoy”, en Nueva Sociedad, No 152.

F. NAISHTAT et al. (2005), Informe de Avance Proyecto UBACyT S090. Buenos Aires, UBA.

F. NEIBURG y M. PLOTKIN (comp.) (2004), Intelectuales y expertos, Buenos Aires, Paidós.

G. O'DONNELL, J. LINZ, E. HOBSBAWN, R. DE JONG (1979), O Estado Autoritária e Os Movimentos

Populares, Conferência sobre História e Ciências Sociais, UNICAMP, Rio de Janeiro, Paz e Terra. 
G. O’DONNELL (1982), 1966-1973 El Estado Burocrático Autoritario, Buenos Aires, Editorial de Belgrano.

A. PÉREZ LINDO (1985), Universidad, politica y sociedad, Buenos Aires, Eudeba.

H. QUIROGA (2004), El tiempo del proceso. Conflictos y coincidencias entre politicos y militares (1976-1983), Rosario, Homo Sapiens Editores / Editorial Ross.

L. ROVELLI (2008), "La impronta modernizadora y los sedimentos de la Ilustración: un estudio del conflicto de proyectos en la UBA durante los años 80 y 90", en F. NAISHTAT y P. ARONSON (comp.), Genealogías de la universidad contemporánea. Sobre la Ilustración o pequeñas historias de grandes relatos, Buenos Aires, Editorial Biblos.

S. SIGAL (1991), Intelectuales y Poder en la década del sesenta, Buenos Aires, Puntosur.

K. SIKKINK (1991), Ideas and Institutions. Developmentalism in Brazil and Argentina, Ithaca and London, Cornell University Press.

T. SKOCPOL (1984), Vision and Method in Historical Sociology, Cambridge, Cambridge University Press.

T. SKOCPOL y M. WEIR (1993), "Las estructuras del Estado: una respuesta keynesiana a la Gran Depresión”, en Zona Abierta, No 63/64.

C. SUASNÁBAR (2004), Universidad e Intelectuales. Educación y Política en la Argentina (19551976), Buenos Aires, FLACSO-Manantial.

O. TERÁN (1991), Nuestros años sesenta, Buenos Aires, Puntosur Editores.

M. C. TORTTI (1999), 'Protesta Social y 'Nueva Izquierda' en la Argentina del Gran Acuerdo Nacional", en A. PUCCIARELLI (editor) La primacía de la política. Lanusse, Perón y la Nueva Izquierda en tiempos del GAN, Buenos Aires, Eudeba.

P. WAGNER; C. WEISS; B. WITTROCK y H. WOLLMAN (1999), Ciencias Sociales y Estados Modernos. Experiencias nacionales e incidencias políticas, México, Fondo de Cultura Económica.

\section{Otras fuentes}

R. ATCON (1966), "La universidad latinoamericana. Propuesta para un enfoque integral de desarrollo social, económico y educacional en América Latina”, Bogotá, Colombia, ECO Revista de la Cultura de Occidente, Ediciones Desalma.

CONADE (1968) Educación, Recursos Humanos y Desarrollo Económico-Social, Tomo I.

CONADE y CONASE (1971) Plan Nacional de Desarrollo y seguridad 1971-1975, Buenos Aires, Presidencia de la Nación.

H. FERRAUTI y A. LENTINI (1978), "La estructura burocrática formal del sector educación de la planta central del Ministerio de Cultura y Educación”, Ediciones de la Revista de Investigaciones Educativas, Buenos Aires.

A. A. LANUSSE (1977), Mi Testimonio, Buenos Aires, Lasserre Editores.

R. MARTORELLI (1991), Crónica de la creación de la Universidad Nacional de Río Cuarto, Río Cuarto, Ed. UNRC.

MINISTERIO DE CULTURA Y EDUCACIÓN (1972) Pautas de creación para universidades nacionales, Buenos Aires.

CONSEJO NACIONAL DE DESARROLlO (1965), Informe sobre el Plan Nacional de Desarrollo1965-1969.

A. C. TAQUINI (1970), “Creación de Universidades”, en Revista Ciencia e Investigación, No 10, pp. $435-450$.

y Cia. Editores. et al. (1972), Nuevas Universidades para un nuevo país, Buenos Aires, A. Estrada

(1984), "Bases para el ordenamiento, jerarquización y expansión de la universidad estatal en el área metropolitana de Buenos Aires" en J. C. AGULLA et al., Universidad y sistema educativo. Libertad y compromiso: convocatoria para un nuevo proyecto de universidad, Buenos Aires, CINAE. 